\title{
A PRESSURE SENSING SYSTEM USING FIBER OPTIC SENSORS AND ARTIFICIAL NEURAL NETWORKS
}

\author{
Xiaomin Jin, Xiao-Hua Yu, Saied Zargar, Roshan H. Patel, and Sam Wards \\ Electrical Engineering Department, 1 Grand Avenue, \\ California Polytechnic State University, San Luis Obispo, CA 93407, USA
}

\section{Introduction}

In the avionic system, it is very important to monitor the pressure difference on the space objective for safety. In this paper, we present a smart pressure sensing system by combining the advanced technologies on fiber optic sensors and computational intelligence to improve the overall sensor system performance. Recently, distributed optical fiber sensor systems have found many applications in civil structural monitoring and power utilities [1]-[3]. An optic fiber is a specially designed medium which can guide the transmission of laser light through its length. When the fiber is bended due to pressure, extra power loss induced by strain will be detected. Unlike the commonly used pressure systems which are susceptible to varying electromagnet fields caused by different sources, fiber sensors are not influenced by EMI (Electromagnetic interference) [4]. In general, fiber sensors are more robust to the disturbances of environment and thus yield better accuracy. Flexible measurement range is another advantage offered by a distributed fiber sensing system, compared with conventional point-sensing methods.

The pressure measurement is actually a complicated nonlinear function with multiple variables of sensor output power and test conditions (e.g., temperature, humidity, etc.). Also, only limited number of measurements can be taken in the experiment. In this research, artificial neural networks are employed to model this relationship. An artificial neural network (ANN) is a biologically inspired computational model which is similar to the real neuronal structure in a human brain. Beyond traditional methods, the neural network based approach has some valuable characteristics, such as the adaptive learning ability, distributed associability, as well as nonlinear mapping ability. Also, unlike conventional approaches, it does not require the complete and accurate knowledge on the system model. Therefore it is usually more flexible when implemented in practice. In recent years, artificial neural networks have received more and more attention and have found many applications in engineering, mathematics, and science [5]- [7].

Multi-layer feed forward neural network employed in this research (Fig. 1) is the class of neural networks that is used most often in dynamical system identification and adaptive control. It has been proved to be very successful in representing any measurable function within any desired degree of accuracy, with the correct values of pressures and sufficient number of hidden neurons.

\section{Conclusion}

The fiber sensor testing system is shown in Fig. 2 (a). Sensors were placed in a hydraulic load machine and connected to the computer. Labview (a software tool) is employed to record the time domain response of sensors. As the machine stepped through various loads (ranging from 0 pounds to approximately 200psi) on the sensor, the output power of sensor is recorded, as shown in Fig. 2 (b). Clearly, the power decreases as the pressure increases, as we expected. The experimental results show the response of the sensor is extremely fast; which indicates a fiber optic sensor would be a very good candidate for pressure detection of objects in the space.

The neural network modeling result is also shown in Fig. 2 (b), where the dotted line represents the measurement data from fiber optic sensors and the solid line represents the output generated by the ANN. From Fig. 2, we conclude that the ANN successfully models the relationship between the sensor output power and the applied pressure. 


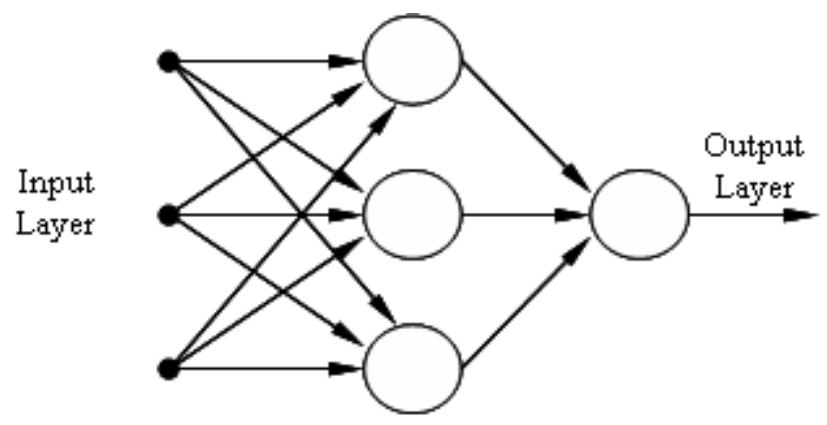

Hidden layer neurons

Fig. 1. Multilayer Feed-forward Network
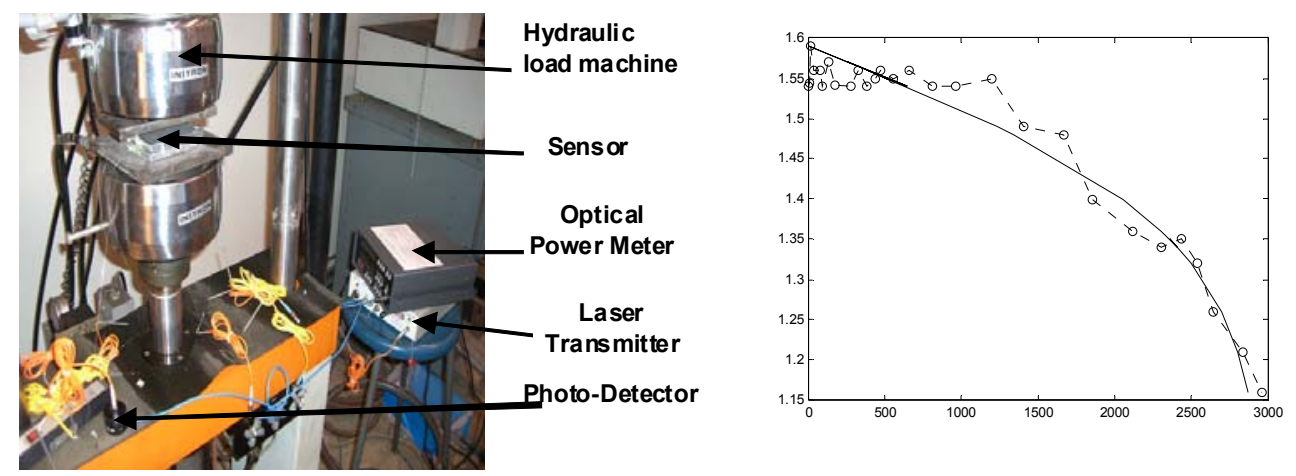

Fig. 2 (a) Fiber sensor test setup; (b) Relationship between the sensor output power (a.u.) and applied pressure: Symbols and the dashed line are the experimental data; the solid line is the neural network result.

\section{References}

[1] T. Hara, K Terashima, H Takashima, H Suzuki, Y Nakura, Y Makino, S Yamamoto, and T Nakamura, "Development of long range optical fiber sensors for composite submarine power cable maintenance", IEEE Transactions on Power Delivery, 14, pp.23-30, 1999.

[2] F.J. Arregui, I.R. Matias, K.L. Cooper, R.O. Claus, "Simultaneous measurement of humidity and temperature by combining a reflective intensity-based optical fiber sensor and a fiber Bragg grating", IEEE Sensors Journal, 2, pp. 482-487, 2002.

[3] V.N. Filippov, A.N. Starodumov, V.P. Minkovich, and F.G.P. Lecona, "Fiber sensor for simultaneous measurement of voltage and temperature", IEEE Photonics Technology Letters, 12, pp. 1543 - 1545, 2000.

[4] B. Degamber and G.F. Fernando, "Process monitoring of a thermosetting resin using optical-fiber sensors in a microwave environment", IEEE Sensors Journal, 4, pp. 713 - 721, 2004.

[5] J.A. Starzyk, Zhen Zhu, and Tsun-Ho Liu, "Self-organizing learning array", IEEE Transactions on Neural Networks, 16, 355- 363, 2005.

[6] S. Ridella, S. Rovetta, and R. Zunino, "K-winner machines for pattern classification", IEEE Transactions on Neural Networks, 12, pp. 371-385, 2001.

[7] Y.-J. Lee and S.-Y. Huang, "Reduced Support Vector Machines: A Statistical Theory", IEEE Transactions on Neural Networks, 18, pp. 1-13, 2007. 